\title{
Losses of runaway electrons during ergodization
}

\author{
K.H. Finken ${ }^{1}$, S.S. Abdullaev ${ }^{1}$, M. Jakubowski ${ }^{1}$, R. Jaspers ${ }^{2}$, \\ M. Lehnen ${ }^{1}$, O. Zimmermann ${ }^{1}$ \\ ${ }^{1}$ Institut für Plasmaphysik, Forschungszentrum Jülich GmbH, EURATOM Association, \\ D-52425 Jülich, Germany \\ ${ }^{2}$ FOM-Institute for Plasma Physics Rijnhuizen, Association EURATOM-FOM, \\ PO Box 1207, 3430 BE Nieuwegein, The Netherlands \\ E-mail: www.rijnh.nl
}

Received 14 July 2005, accepted for publication 20 October 2005

Published 15 March 2006

Online at stacks.iop.org/NF/46/S139

\begin{abstract}
The dynamic ergodic divertor (DED) of TEXTOR has been applied to runaway discharges. The runaway electrons $(\varepsilon<30 \mathrm{MeV})$ are developed as probes for investigating the internal magnetic field line structure in the plasma. Complementary diagnostics are used, namely neutron measurements for the loss of the runaways and synchrotron radiation for the detection inside the plasma. During the DED phase, three features of the runaway electrons are found: a sudden loss from the just formed ergodic layer, an enhanced diffusive transport and very sudden loss events which may be related to field line reconnection processes.
\end{abstract}

PACS numbers: 52.35.- g, 52.27.Ny, 52.35.Vd

\section{Introduction}

In this paper runaway electrons are employed to investigate the transport in plasma regions which are dominated by ergodized magnetic field lines generated by the dynamic ergodic divertor (DED) of TEXTOR.

The phenomenon of electron runaway results from the fact that the mean free path of an electron in the plasma is a strongly decreasing function of its velocity. In an electric field, electrons exceeding a critical velocity for which the collisional drag balances the acceleration by the field are accelerated freely and can reach very high energies. At these energies they are effectively collisionless and follow (apart from their systematic displacement to the low field side (LFS), see section 5) the magnetic field lines. Therefore they provide a unique tool to probe the magnetic field perturbations.

Runaway electrons have been observed in nearly all tokamaks, mainly during disruptions (due to the high induced electric field), where they can reach energies of several tens of megaelectronvolts [1-3]. They are also encountered in other fields of plasma physics, e.g. in solar flares or thunderstorms [4]. The runaways can cause severe damage to first wall components in particular during disruptions because they are deposited very locally and reach extremely high energies [5].

Over the last decade, runaway studies have been performed at TEXTOR. These experiments were unique in the fact that the synchrotron radiation of relativistic electrons in the energy range $20-30 \mathrm{MeV}$ was exploited for observing confined runaway electrons. These measurements yielded a wealth of information on runaway electron generation [6,7], the runaway electron transport [8-11] and on the possibility of visualizing drift orbit islands of runaway electrons $[12,13]$.

\section{Runaway diagnostics}

The synchrotron radiation provides a direct image of the runaway beam inside the plasma, recorded with a thermographic camera. From the spectral features the runaway energy can be obtained; the intensity of the radiation is a measure of the number of runaway electrons, and the synchrotron spot carries information on their perpendicular momentum and spatial distribution. Detailed information on the synchrotron emission by relativistic electrons and the diagnostic can be found in [14]. The required electron energy for the generation of synchrotron radiation amounts to about $\varepsilon=25 \mathrm{MeV}$ which requires a free fall time of about $1 \mathrm{~s}$ at a loop voltage of $1 \mathrm{~V}$. The typical runaway current in 'normal' low density discharges $\left(n_{\mathrm{e}}<1 \times 10^{19} \mathrm{~m}^{-3}\right)$ TEXTOR is $5-10 \mathrm{kA}$ at a plasma current of typically $350 \mathrm{kA}$.

An alternative, more often used method for detecting runaway electrons is the measurement of hard x-rays or neutrons; both the x-rays and the neutrons are predominantly 


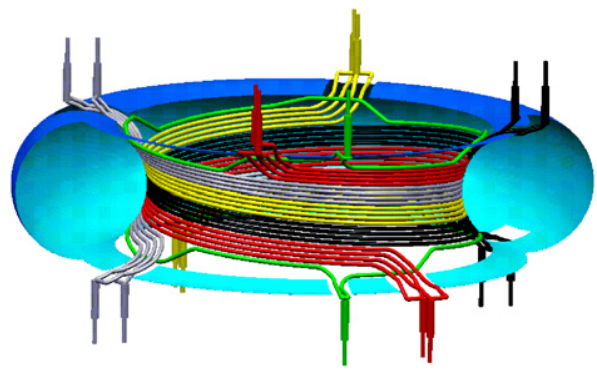

$\mathrm{m}=3, \mathrm{n}=1$

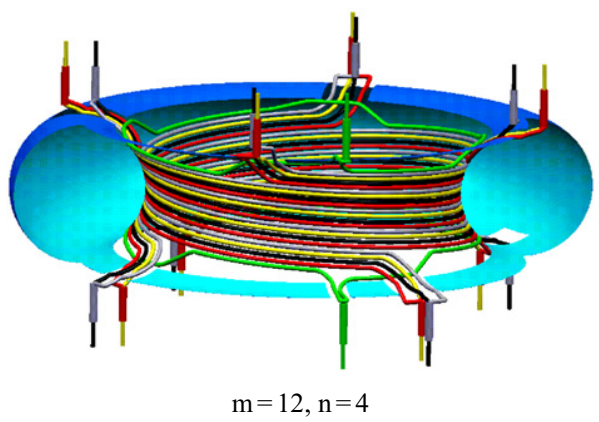

Figure 1. Sketch of the $m / n=12 / 4$ and $3 / 1$ base configurations of the DED.

(This figure is in colour only in the electronic version)

created when the relativistic electrons have left the plasma and hit wall components. Therefore synchrotron radiation and the measurements of $\mathrm{x}$-rays/neutrons are complementary, the first one giving the runaways inside the plasma and the second one giving the loss rate.

\section{The dynamic ergodic divertor}

Its main component is a system of 16 coils wound helically around the torus at the high field side inside the vacuum vessel. The winding follows essentially the magnetic field lines near the $q=3$ surface. Electrical currents flowing in the coils generate a perturbation field nearly perpendicular to the equilibrium field and result at a sufficient perturbation current level in an ergodization of the magnetic field lines.

Since all 16 DED-coils are fed to the outside of the torus, it is possible to use different interconnections. In this context we apply (a) the so called $m / n=3 / 1$ base mode in which 4 neighbouring coils are switched in parallel and the neighbouring bundles are fed with currents of a phase difference of $90^{\circ}$ and (b) the $m / n=12 / 4$ base mode in which all neighbouring coils are fed with currents of a phase difference of $90^{\circ}$. Figure 1 shows a sketch of the two scenarios. It is obvious that in case (a) the poloidal current distribution is much coarser than in case (b) and therefore the field penetrates radially substantially deeper into the plasma. In case (a) the field penetrates practically to the plasma centre while in case (b) the perturbation is restricted to the edge only. It has been shown that the deep penetration of the perturbation field (a) leads to the excitation of $m / n=2 / 1$ and $m / n=3 / 1$ tearing modes if specific threshold values of the DED current are exceeded $[15,16]$. Because of the very shallow field penetration, the $m / n=12 / 4$ base mode configuration of the DED does not show the tendency to excite tearing modes.

\section{Results}

\subsection{Observations during the $m / n=3 / 1$ base mode}

A typical runaway electron discharge with DED in the $m / n=$ $3 / 1$ base configuration is shown in figure 2 . The decisive parameter for obtaining a runaway discharge is the low electron density as shown in $(b)$. The density is kept constant to $0.6 \times 10^{19} \mathrm{~m}^{-3}$; this choice guarantees a continuous generation and acceleration of the runaways during the discharge. The discharge shows frequent sawtooth activity (typically $10 \mathrm{~ms}$ per period) independent of the application of the DED; the activity is also not altered when the later described runaway crashes occur. In this phase, two different generation processes occur: the first, which we refer to as primary generation, treats the diffusion in velocity space of the electron distribution around the critical velocity. The second mechanism, secondary generation, is the process in which already existing high energetic electrons kick thermal electrons into the runaway regime by close Coulomb collisions. The production rate in this case is proportional to the number of runaway electrons and is therefore expected to lead to an exponential growth rate of the runaway generation [17]. Because of this it is often referred to as the avalanche mechanism. After about $2 \mathrm{~s}$ the runaways emit sufficient synchrotron radiation such that they can be distinguished from the thermal background. The maximum energy of the runaway electrons present at this time is estimated to be $25-30 \mathrm{MeV}$, at which energy the emission is balanced by the energy gained from the electric field.

The measurement with the application of the DED perturbation field is performed in the interval $3 \mathrm{~s}<t<4 \mathrm{~s}$. After $4 \mathrm{~s}$ the electron density is raised by helium injection; this injection reduces the wall activation from the runaways by an order of magnitude, since the runaways are decelerated in the plasma core.

At $3 \mathrm{~s}$ the DED is switched on for $1 \mathrm{~s}$ as shown in figure 2(a); the DED current is kept at a constant level in this time interval. The ECE signal- $(d)$-is normally used for the determination of the electron temperature. In runaway discharges, the emission is non-thermal and obviously governed by the low-energy part of the distribution function of the runaway electrons. In contrast to the usual ECE, the emission frequency cannot be attributed to a radial location because (a) the synchrotron radiation has a continuous spectrum, (b) the relativistic mass increment $\gamma$ shifts the locus of emission and (c) the plasma is not optically thick everywhere for these frequencies.

The spectrum of the synchrotron radiation had been discussed in [3]: at low frequencies, in the microwave range, the individual low and high energy runaway electron contributes asymptotically with the same weight to the spectrum. At higher frequencies, the low energy electrons stop successively contributing to the spectrum. If the number of low energy electrons exceeds the one of high energy runaways, the microwave emission is dominated by the low energy part. The observed ECE signal is about an order of magnitude higher than the thermal one and saturates several ECE channels. The 

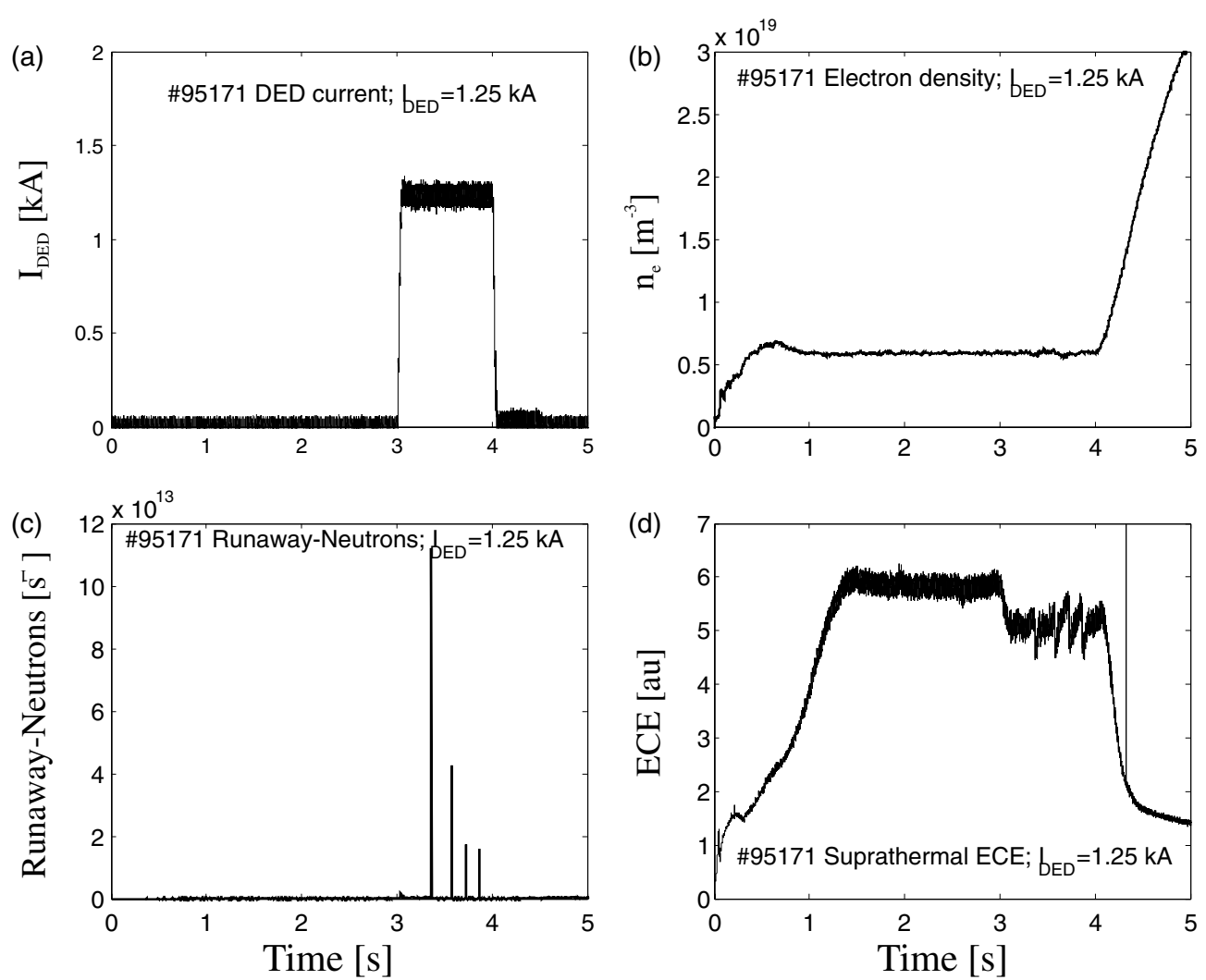

Figure 2. Typical traces during runaway experiments with DED. (b) Corresponds to the density trace, $(a)$ to the DED current, $(d)$ to the ECE emission signal and $(c)$ to the neutron signal.

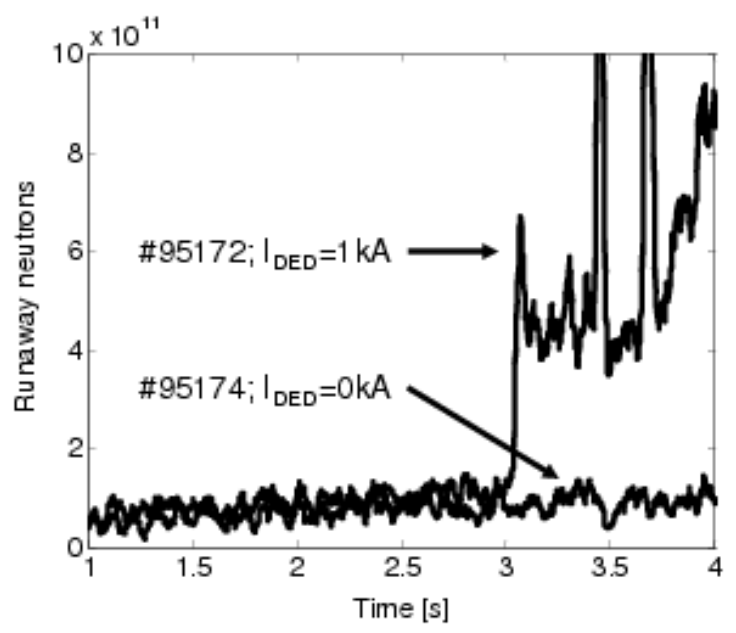

Figure 3. Neutron signal for a discharge without DED (blue) and another one with DED (red; DED on from $3 \mathrm{~s}<t<4 \mathrm{~s}$ ).

non-saturated channels show an increase of the ECE-signal up to $1.3 \mathrm{~s}$ which then becomes constant; this trend is in contrast to the high energy signature which shows an increase over the whole discharge (compare figure 4).

With the onset of the DED at $3 \mathrm{~s}$ one sees a sudden drop of the ECE signal to a new level. This level is interrupted by sudden loss events which last only a short time. The sudden loss events are more distinctively seen in the spikes of the neutron signal (figure 2(c)) which result from the runaway electrons hitting the wall components. These spikes are observed only in the $m / n=3 / 1$ base configuration of the DED and are linked with a substantial loss of the runaways.

Figure 3 shows the neutrons resulting from the runaway loss in higher magnification for a discharge with DED (top curve) and for a reference discharge without DED; the DED current is rather modest with $1 \mathrm{kA}$. The signals are practically identical before the onset of the DED at $3 \mathrm{~s}$. With the onset of the DED at first a transient spike is observed followed by a phase with a factor of 3 higher loss rate than without DED. Finally one or more prominent large spikes are observed. We attribute the first spike to the formation of the ergodic layer and the neutron pulse is interpreted as the rapid loss from this area. We assume that the second phase of the runaway loss rate (as seen by the increased neutron signal) is caused by deep reaching perturbation fields which however are not sufficient to cause ergodization of the magnetic field lines. Therefore the runaway electrons should not be lost immediately by open field lines to the wall which would be a very fast process because of the high velocity of the runaways. Instead, we interpret the steady state loss as an enhanced diffusion. The large spikes are not understood; tentatively we explain them as reconnection events of the magnetic field due to the perturbation.

The complementary measurement to the neutron loss rate is the synchrotron radiation from the runaways inside the plasma. Figures $4(a)$ and $(b)$ give the time traces of the synchrotron radiation for different levels of the DED current. Figure 4(a) shows data for low DED currents $I_{\mathrm{DED}} \leqslant 1.25 \mathrm{kA}$, 

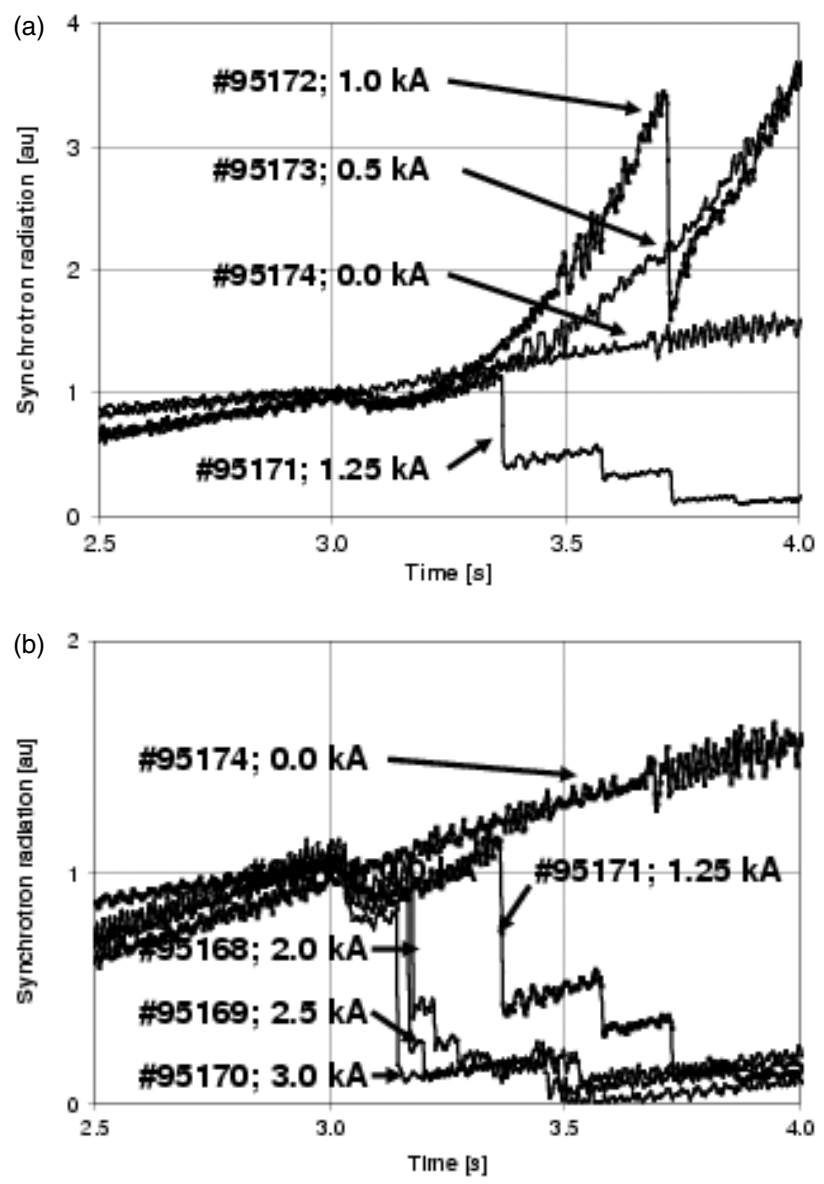

Figure 4. (a) Shows the synchrotron radiation in arbitrary units for 'low' DED current, while $(b)$ gives similar curves for 'high' DED current. For reference both figures contain the data for $I_{\mathrm{DED}}=0 \mathrm{kA}$ and $I_{\mathrm{DED}}=1.25 \mathrm{kA}$. The data refer to data in the $m / n=3 / 1$ base mode.

while figure 4(b) exhibits the higher DED currents. In both figures, the discharge number and the applied DED currents are indicated.

Without DED, the synchrotron signal increases slowly in time because the number of runaways increases. All other curves show an initial drop of the radiations at $3.0 \mathrm{~s}$ when the DED is switched on. This loss of runaway electrons measured by the synchrotron radiation corresponds to the loss measured by the neutrons. At $t=4$ we inject helium as mentioned before. This leads to a gradual decrease of the synchrotron radiation but is not depicted here.

At 'low' DED currents $\left(I_{\mathrm{DED}}=0.5\right.$ and $\left.1.0 \mathrm{kA}\right)$, the initial drop is overcompensated by a growth of the synchrotron radiation, which is substantially larger than in the ohmic phase. The increase can result from the increasing number of runaways, from their energy or from their radius of curvature; since the loop voltage increases typically from $0.8 \mathrm{~V}$ in the ohmic phase to $1.0 \mathrm{~V}$ in the DED phase, we assume that the particle number and the energy grow. The increase in number is corroborated by the rise of the neutron signal in this phase.

Remarkable are the sudden runaway loss events at increased DED current. Both the number and the loss per event increase with increasing DED perturbation current. The loss is so substantial that at $I_{\mathrm{DED}}>2 \mathrm{kA}$ practically all runaways are
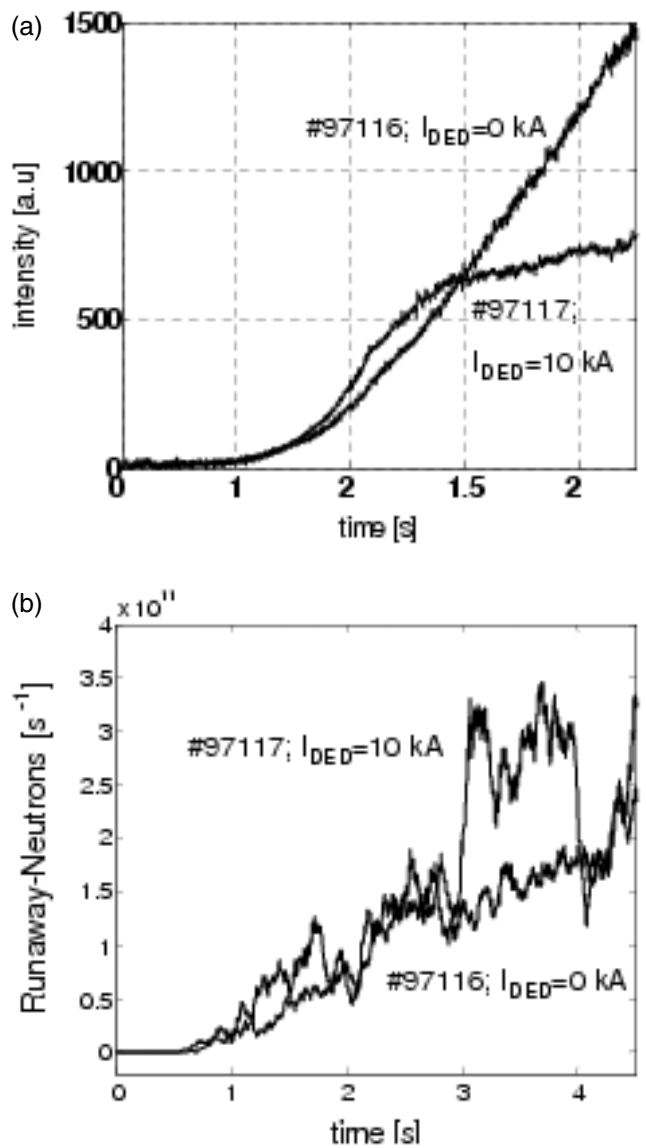

Figure 5. Synchrotron intensity $(a)$ and neutron signals $(b)$ as a function of time. The discharge numbers and DED currents are indicated.

expelled. The loss events correspond in all cases to the large neutron spikes and thus provide consistent complementary results.

\subsection{Observations during the $m / n=12 / 4$ base mode}

So far, the results correspond to the operation with the $m / n=$ $3 / 1$ base mode. It is therefore of particular interest to compare the runaway loss at a deeply penetrating perturbation field with the perturbation field restricted to the plasma edge of the $12 / 4$ base mode. Figures $5(a)$ and $(b)$ show the synchrotron intensity $(a)$ and neutron signals $(b)$ as a function of time. The discharge numbers and DED currents are indicated; the DED is again applied in the interval $3 \mathrm{~s}<t<4 \mathrm{~s}$. The discharges were not exactly reproducible and therefore the neutron signal without DED is in the reference non-DED phase slightly smaller than during the DED phase; therefore the signal is re-normalized by an appropriate multiplication factor.

Even though the DED current is quite substantial, its effect on the runaway loss is rather mild. The neutron signal has a shape which is just proportional to the DED current without the initial spike and the large bursts as discussed in the previous section. The loss rate in the neutron signal just doubles and is nearly as high as in the example of figure 2 . The synchrotron radiation is consistently reduced when the DED is switched on; however, the loss rate is rather modest, smooth and without any sudden events. 


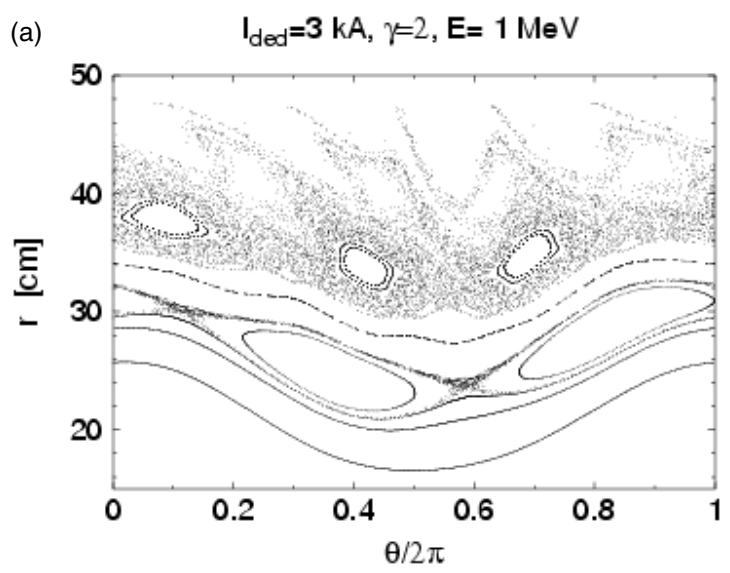

(b)

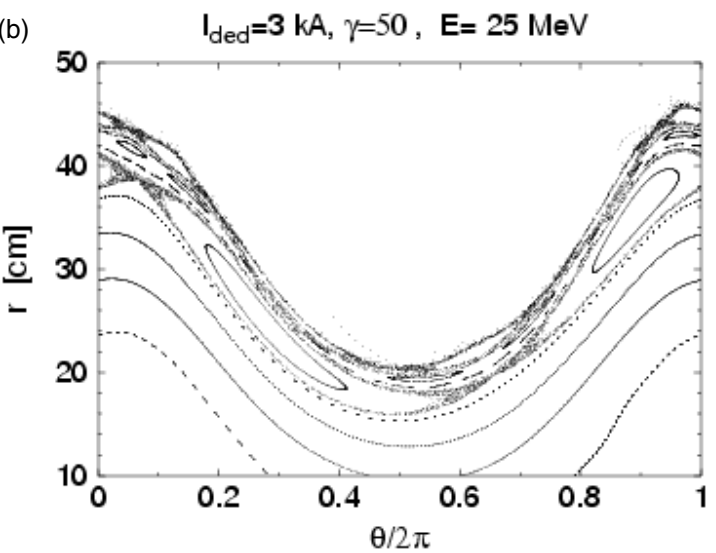

Figure 6. Poincaré sections of electron orbits with an energy of $1 \mathrm{MeV}(a)$ and of $25 \mathrm{MeV}(b)$. In both figures, the abscissa is the poloidal angle and the ordinate is the minor radius. The data are obtained from guiding centre tracing at a DED-current of $3 \mathrm{kA}$.

\section{Theoretical analysis}

Ergodization of runaway electrons in the presence of external magnetic perturbations is completely different from the ergodization of magnetic field lines since due to toroidal drifts particle orbits differ from the magnetic field lines. In tokamaks the runaway electrons move in the direction opposite to the plasma current, i.e. they are counter passing particles. It is known that the orbits of these particles are shifted towards the LFS. In typical TEXTOR conditions the orbit shift on the HFS is $10-15 \mathrm{~cm}$ for runaway electrons of energy $20 \mathrm{MeV}$. Since the DED perturbation field is poloidally localized on the HFS and it decays radially one expects that the effect of the DED on runaways will be insignificant in the case of $m / n=12 / 4$ mode. The modest influence of the DED on runaways occurs in the $m / n=3 / 1$ mode operation when the perturbation field deeply penetrates into the plasma.

For the quantitative analysis of the dynamics of runaway electrons we have derived the Hamiltonian guiding centre equations for relativistic particles by generalizing the method proposed in [18]. These Hamiltonian equations of runaway electrons are integrated using the mapping approach [19].

Two results of the mapping are shown in figures $6(a)$ and $(b)$. They show Poincaré plots of electron orbits on the poloidal section at an energy of $1 \mathrm{MeV}(a)$ and at $25 \mathrm{MeV}(b)$ in the $m / n=3 / 1$ operational mode. In both figures, the
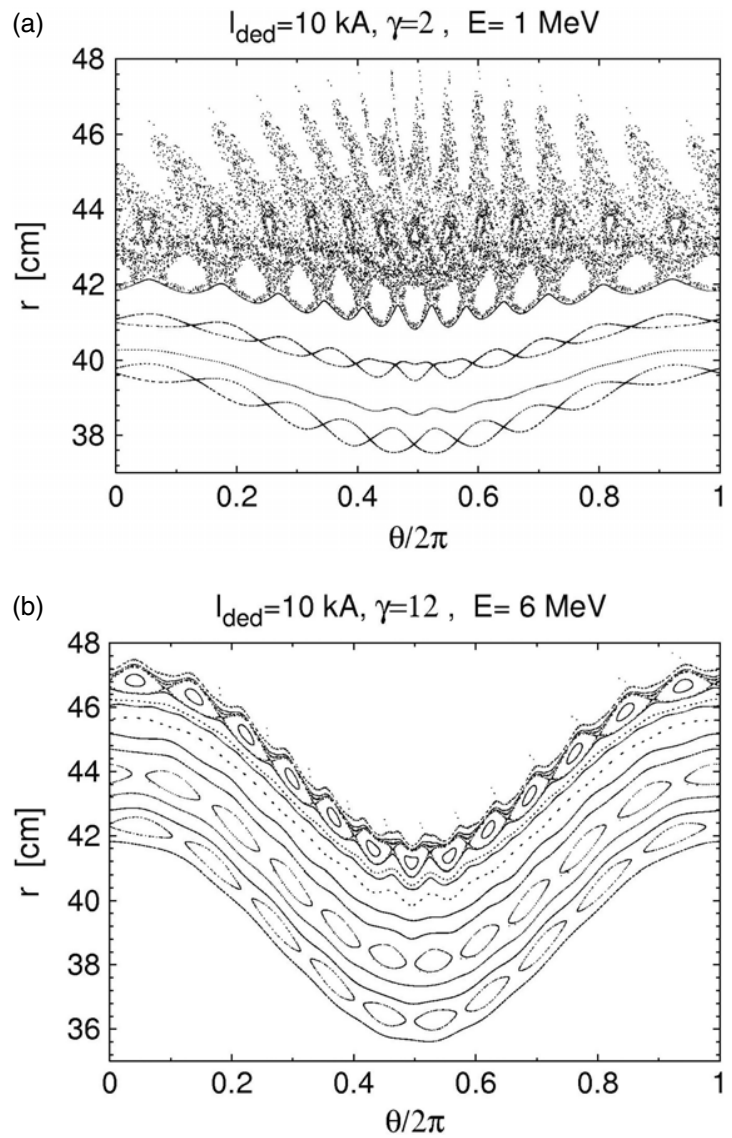

Figure 7. The same as in figures $6(a)$ and $(b)$, but in the $m / n=12 / 4$ mode. (a) This figure describes the electrons with an energy $1 \mathrm{MeV}$ and $(b)-6 \mathrm{MeV}$. The plasma and DED parameters correspond to the shot \#9711 (see figure 5).

abscissa is the poloidal angle and the ordinate is the minor radius. The data are obtained from guiding centre tracing of the runaway electrons at a DED-current of $3 \mathrm{kA}$. It is obvious that the electrons with lower energy can populate a much wider area of the torus than those with the higher energy. Because the ergodic areas are finally connected to the vessel wall, it is expected that the ergodized areas are quickly depopulated. The depopulation of the runaway electrons is much more effective than for thermal particles because they move practically at the speed of light and they undergo no collisions which reduces their loss. In the case of the $m / n=12 / 4$ mode the effect of the DED on runaway electrons is less pronounced. Figures $7(a)$ and $(b)$ show the corresponding Poincaré plots of electron orbits with an energy $1 \mathrm{MeV}(a)$ and $6 \mathrm{MeV}(b)$; in $(b)$ we have used the relative low value of $6 \mathrm{MeV}$ because at the full runaway energy the effect of the ergodization on the runaway orbits vanishes. As seen from these figures the area of ergodized electrons due to the DED decreases with increasing electron energy. For electrons with an energy less than 1-3 MeV the ergodized area is close to the one of magnetic field lines. The electrons with energy higher than $6 \mathrm{MeV}$ are practically not affected by the DED. The enhanced loss rate of figure $5(b)$ during the application of the DED is interpreted as loss of the electrons with lower relativistic energy, e.g. $\varepsilon<10 \mathrm{MeV}$, which experience the ergodization. Therefore the synchrotron radiation is not diminished; however, its growth is 
substantially reduced because the reservoir of lower energetic particles is depleted which otherwise would develop to high energetic runaways.

\section{Discussion}

The energetic runaway electrons are very interesting probes for investigating the magnetic field pattern perturbed by the DED and the resulting transport. For these investigations, two diagnostics are available: (a) the measurements of the runaway loss by hard x-rays or runaway induced neutrons and (b) the detection of runaway electrons inside the plasma by synchrotron radiation. Both diagnostics are complementary and provide consistent results. It is found that the perturbation field penetrates very quickly into the plasma and that in the $\operatorname{DED} m / n=3 / 1$ base mode a relative wide area of runaway electrons is depopulated; the signature of this depopulation appears as a first spike in the neutron signal. In the DED $m / n=12 / 4$ base mode, this area is considerably smaller and therefore no pronounced loss peak is observed.

After the first loss spike one observes an enhanced diffusion which is attributed both to the smaller remaining confinement volume after the ergodization and to deep reaching perturbation fields. The quasi-steady diffusive transport is about $50 \%$ higher in the DED $m / n=3 / 1$ base mode than in the $12 / 4$ mode. This result is consistent with the deeper penetration of the coarse $3 / 1$ base mode.

Not well understood up to now are the strong bursty losses which are observed in the DED $m / n=3 / 1$ base mode only. This indicates that the events occur deeply inside the plasma at radii which are not reached by the $12 / 4$ mode. Tentatively we explain the bursts as reconnection events of the magnetic field lines. However, it is not understood on which instability the process is based.
It was stated before that the synchrotron radiation allows the locally resolved detection of runaways inside the plasma. It was indeed one of the aims to investigate the area from where the runaway electrons are preferentially lost. Unfortunately, we had technical difficulties with the IR camera during the campaign such that we could see only a very limited part of the plasma cross section. It is required to re-optimize the IR system such that a better view is possible and the nature of the sudden spikes can be unveiled.

\section{References}

[1] Knoepfel H. and Spong D.A. 1979 Nucl. Fusion 19785

[2] Gill R.D. et al 2000 Nucl. Fusion 40163

[3] Finken K.H. et al 1990 Nucl. Fusion 30859

[4] Gurevich A.V. et al 1996 Radio Sci. 311541

[5] Putvinski S. et al 1997 Plasma Phys. Control. Fusion 39 B157

[6] Jaspers R. et al 1993 Nucl. Fusion 331775

[7] Pankratov I.M. et al 1998 Nucl. Fusion 38279

[8] Jaspers R. et al 1996 Nucl. Fusion 36367

[9] Jaspers R. et al 1995 J. Nucl. Mater. 220-222 682

[10] Entrop I., Lopes Cardozo N.J., Jaspers R. and Finken K.H. 1998 Plasma Phys. Control. Fusion 401513

[11] Entrop I., Lopes Cardozo N.J., Jaspers R. and Finken K.H. 2000 Phys. Rev. Lett. 843606

[12] Jaspers R. et al 1994 Phys. Rev. Lett. 724093

[13] Entrop, Jaspers R., Lopes N.J. Cardozo and Finken K.H. 1999 Plasma Phys. Control. Fusion 41377

[14] Jaspers R. et al 2001 Rev. Sci. Instrum. 72466

[15] Finken K.H. et al 2005 Phys. Rev. Lett. 94 015003-1

[16] K H Finken et al 2004 Plasma Phys. Control. Fusion 46 B143

[17] Rosenbluth M.N. and Putvinski S.V. 1997 Nucl. Fusion 37 1355

[18] Abdullaev S.S. and Finken K.H. 2002 Phys. Plasmas 94193

[19] Abdullaev S.S., Finken K.H. and Spatschek K.H. 1999 Phys. Plasmas 6153 